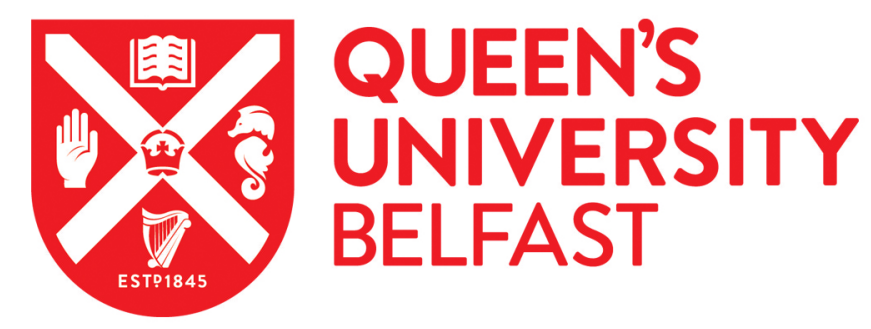

\title{
Indexing and matching trajectories under inconsistent sampling rates
}

Ranu, S., Padmanabhan, D., Telang, A. D., Deshpande, P., \& Raghavan, S. (2015). Indexing and matching trajectories under inconsistent sampling rates. In Proceedings of the 2015 IEEE 31st International Conference on Data Engineering, ICDE 2015 (pp. 999-1010). Institute of Electrical and Electronics Engineers Inc.. https://doi.org/10.1109/ICDE.2015.7113351

Published in:

Proceedings of the 2015 IEEE 31st International Conference on Data Engineering, ICDE 2015

\section{Document Version:}

Peer reviewed version

\section{Queen's University Belfast - Research Portal:}

Link to publication record in Queen's University Belfast Research Portal

\section{Publisher rights}

( 2015 IEEE. Personal use of this material is permitted. Permission from IEEE must be obtained for all other uses, in any current or future media, including reprinting/republishing this material for advertising or promotional purposes, creating new collective works, for resale or redistribution to servers or lists, or reuse of any copyrighted component of this work in other works.

\section{General rights}

Copyright for the publications made accessible via the Queen's University Belfast Research Portal is retained by the author(s) and / or other copyright owners and it is a condition of accessing these publications that users recognise and abide by the legal requirements associated with these rights.

Take down policy

The Research Portal is Queen's institutional repository that provides access to Queen's research output. Every effort has been made to ensure that content in the Research Portal does not infringe any person's rights, or applicable UK laws. If you discover content in the Research Portal that you believe breaches copyright or violates any law, please contact openaccess@qub.ac.uk. 


\title{
Indexing and Matching Trajectories under Inconsistent Sampling Rates
}

\author{
Sayan Ranu ${ }^{\# 1}$, Deepak $\mathrm{P}^{* 2}$, Aditya D. Telang ${ }^{* 2}$, Prasad Deshpande ${ }^{* 2}$, Sriram Raghavan ${ }^{* 2}$ \\ \# Dept. of CSE, IIT Madras, Chennai, India. \\ * IBM Research, Manyata Tech. Park, Bangalore, India. \\ ${ }^{1}$ sayanecse.iitm.ac.in \\ ${ }^{2}$ \{deepak.s.p, aaditya.telang, prasdesh, sriramraghavan\}@in.ibm.com
}

\begin{abstract}
Quantifying the similarity between two trajectories is a fundamental operation in analysis of spatio-temporal databases. While a number of distance functions exist, the recent shift in the dynamics of the trajectory generation procedure violates one of their core assumptions; a consistent and uniform sampling rate. In this paper, we formulate a robust distance function called Edit Distance with Projections $(E D w P)$ to match trajectories under inconsistent and variable sampling rates through dynamic interpolation. This is achieved by deploying the idea of projections that goes beyond matching only the sampled points while aligning trajectories. To enable efficient trajectory retrievals using EDwP, we design an index structure called TrajTree. TrajTree derives its pruning power by employing the unique combination of bounding boxes with Lipschitz embedding. Extensive experiments on real trajectory databases demonstrate EDwP to be up to 5 times more accurate than the state-of-the-art distance functions. Additionally, TrajTree increases the efficiency of trajectory retrievals by up to an order of magnitude over existing techniques.
\end{abstract}

\section{INTRODUCTION}

The last decade has witnessed an unprecedented growth in the availability of location-tracking devices. The widespread usage of these devices generates an abundance of data that are in the form of trajectories. Querying and mining these trajectories is essential for a multitude of spatio-temporal tasks such as tracking migratory patterns of animals [1], identifying "alleys" conducive to environmental disasters [2], etc. What lies at the core of any of these analytical tasks is a mechanism to compute the similarity between two trajectories. The importance of matching trajectories has been recognized in the computer science community and a number of techniques exist [3][7]. However, the recent explosion in the volume of trajectory data is fueled by the availability of cheap and heterogeneous location tracking devices that violate an assumption made by all of the existing trajectory matching techniques; the assumption of a uniform and consistent sampling rate. In this paper, we study the problem of matching trajectories in the presence of this sampling noise.

Generally, a trajectory $T=\left[s_{1}, \cdots, s_{n}\right]$ of a moving object is represented as a sequence of spatio-temporal points $s_{i}$ that are sampled over a time duration. A spatio-temporal point $s=(x, y, t)$ encodes the spatial-attributes of the location, such as latitude and longitude, and the timestamp $t$ at which the location was traversed. Two trajectories are considered similar if they remain spatially close to each other for the majority of their existence. A basic model to match trajectories is through the $L^{p}$-norm and create a one-to-one alignment between the sampled points. This model, however, suffers in cases of local time shifts. Consider two trajectories that traverse the same spatial contour, where one of them is slower in the first half of the distance, and the other is slower in the second half. At an uniform sampling rate, the first trajectory would have more points in the first spatial half than the second. Consequently, the true distance would not be captured. Dynamic Time Warping (DTW) [6] first recognized this issue and accounted for local time shifts using many-toone mappings. Subsequently, DTW was further improved by better capturing the spatial semantics in Edit distance with Real Penalty (ERP) [4], Edit Distance on Real sequence (EDR) [5], DISSIM [7], and model-driven assignment (MA) [8].

While existing techniques can adapt to local time shifts, they are unable to cope well with non-uniform sampling rates. In real life, there are variations in sampling rates within and across trajectories induced through variable device settings, power constraints, intermittent signal disruptions, etc. For example, a recent study has shown that cab drivers alter the default sampling rate in their GPS-navigation systems to reduce power consumption [9]. Furthermore, sampling rates in trajectories generated from sources such as online "checkins" (eg. FourSquare), gps-tagged photo albums, and call detail records are inherently non-uniform. These variations in inter-trajectory as well as intra-trajectory sampling rates pose a number of unique challenges that are not yet adequately addressed. To combat these issues, we make the following contributions in trajectory matching:

- We develop a robust distance function, called Edit Distance with Projections (EDwP), to compute similarity between trajectories. EDwP employs a threshold-free approach and automatically adapts to non-uniform sampling rates through dynamic interpolation by using the idea of projections.

- We develop an index structure called TrajTree for efficient retrieval of $k$-NN queries. The proposed index structure combines the ideas of Lipschitz embedding [10] with bounding boxes [11] to drastically prune the search space.

- Extensive experiments performed on real datasets show EDwP as up to 5 times more accurate and robust than state-of-the-art trajectory matching techniques. Furthermore, TrajTree is efficient in pruning the search space, which results in an order of magnitude speedup over the most recent trajectory matching technique [8] and 5X speedup over EDR [5]. 


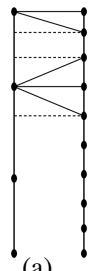

(a)

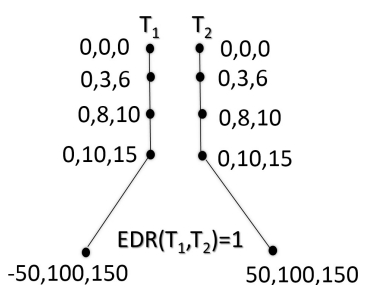

(b)

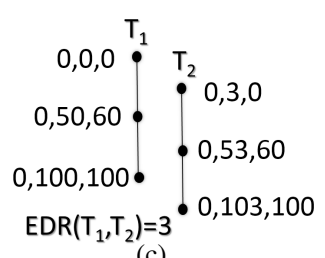

(c)

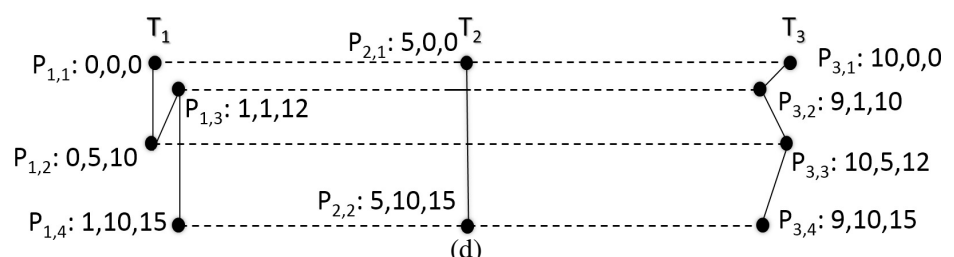

(d)

Fig. 1. Illustrates the weaknesses of existing techniques using EDR [5] as the representative metric against (a) inter-trajectory sampling rate variance, (b) intra-trajectory sampling rate variance and (c) "phase" shifts. The first two dimensions in each st-point represents the spatial location, and the third dimension represents the timestamp at which the location is recorded. The distances computed using EDR assumes a spatial threshold of $\epsilon=2$. (d) Illustrates a scenario where the mapping produced by MA [8] is not logical.

\section{IMPACT OF INCONSISTENT SAMPLING RATES}

In this section, we analyze the impact of varying sampling rates on existing trajectory matching techniques.

1. Inter-trajectory Sampling Rate Variations: Consider Fig. 1(a) where the two trajectories represented by the vertical lines are being compared; the left one is sparsely sampled (only 4 points) whereas, the right one has a higher rate of sampling. Since most existing techniques (except DISSIM [7] and MA [8]) start by mapping sampled points between the trajectories, the best mappings include those as indicated by the slanted solid lines joining points across trajectories, which cause a larger spatial distance between mapped points than the more intuitive mappings indicated by the horizontal dotted lines.

MA [8] is more flexible in aligning points. While trying to align a sampled point $p_{1}$ in trajectory $T_{1}$ to another sampled point $p_{2}$ in $T_{2}$, it considers any non-sampled point in the straight line connecting $p_{2}$ to the last aligned point in $T_{2}$ prior to $p_{2}$. Fig. 1(d) illustrates an example. This technique, however, may produce alignments that are semantically inconsistent. Specifically, in Fig. 1(d), $p_{1,2}$ and $p_{1,3}$ in $T_{1}$ get mapped to two non-sampled points in $T_{2}$. Here, $p_{1,3}$ is mapped to a point that is traversed prior to the point where $p_{1,2}$ is mapped. In other words, it introduces alignments that goes backward in time, and thus, violates the basic premise of time-series matching. Consider another trajectory $T_{3}$ in the figure that is formed by points that are as far away from $T_{2}$ as those of $T_{1}$; the difference in the order of traversing points among $T_{1}$ and $T_{3}$ intuitively makes $T_{3}$ much more similar to $T_{2}$ than $T_{1}$. However, MA relies on aggregating the distance between matched points, and thus spuriously assigns the same similarity for the $\left(T_{1}, T_{2}\right)$ pair as for $\left(T_{2}, T_{3}\right)$.

DISSIM also incorporates non-sampled regions in the distance computation. However, DISSIM cannot cope with local time shifts due to only considering one-to-one mappings. Thus, DISSIM can detect similarity between trajectories under nonuniform sampling rates only if they travel at identical speeds.

2. Intra-trajectory Sampling Rate Variations: Besides inter-trajectory sampling rate variation, sampling rates also vary within a trajectory. This results from signal disruptions, power constraints, etc. Fig. 1(b) illustrates the issue by taking one of the most recent works, EDR, as the representative metric. EDR considers two points to "match" if they are within a spatial distance threshold $\epsilon$. Let us assume $\epsilon=2$. As can be seen, four out of the five points are identical and accordingly, EDR assigns a distance of 1 due to the dissimilarity between the fifth points. However, all of the four matched points represent a densely sampled region and in reality, for the majority of the two trajectories, they diverge from each other. As a result, a distance of 1 is not an accurate reflection of the significant dissimilarity existing between them.

One could certainly tackle varying sampling rates by interpolating points in the under-sampled regions. Such an approach however, suffers from three bottlenecks. First, to ensure a uniform sampling rate, interpolation should be performed such that the processed database of trajectories have a uniform density that is equal to the maximum density observed in the unprocessed

If the trajectories are constrained within a road network, one could consider map-matching [12], [13]. However, it has been shown that at low sampling rates, map-matching is not accurate [14]. More critically, many trajectories such as animal migrations, ship and airplane movements, pedestrian trajectories, etc. are not constrained within a network.

3. Sampling "Phase" Variations: Since st-points only represent a sample of the locations traversed, the choice of recorded samples can have a drastic impact on the computed distance even under uniform sampling. Fig. 1(c) depicts two trajectories that are sampled uniformly from their starting points. Although $T_{1}$ and $T_{2}$ overlap for the majority of their existence in the spatial region between $(0,3)$ and $(0,100)$, they are not represented by the same set of sampled points. As a result, under a spatial threshold of $\epsilon=2$ for EDR (or LCSS), none of the st-points match, and consequently, the maximum possible distance of 3 is assigned.

4. Threshold Dependency: EDR, ERP and LCSS, use thresholds to determine similarity between locations. MA depends on four different thresholds. Generally, thresholddependent techniques grid the physical space into regions, and two spatial locations "match" if they fall within the same grid. Determining the appropriate gridding threshold, however, is not straightforward and can have a large impact on the similarity. For example, in Fig. 1(c), at $\epsilon=2, \operatorname{EDR}\left(T_{1}, T_{2}\right)$ assigns the maximum possible distance of 3 . On the other hand, a small increase of $\epsilon=3$ reduces $\operatorname{EDR}\left(T_{1}, T_{2}\right)$ to the lowest possible distance 0 .

Table I summarizes the features of six of the most recent distance metrics. In this paper, our goal is to answer all of the outlined challenges without compromising on the positive aspects of any of the existing distance functions. 
TABLE I. Robustness of Previous Trajectory Similarity METRICS

\begin{tabular}{|c|c|c|c|c|c|}
\hline \multirow[t]{2}{*}{ Technique } & Local & \multicolumn{3}{|c|}{ Sampling Rate Variations } & Threshold \\
\hline & $\begin{array}{l}\text { Time } \\
\text { Shifts }\end{array}$ & $\begin{array}{c}\text { Inter- } \\
\text {-trajectory }\end{array}$ & $\begin{array}{c}\text { Intra- } \\
\text { trajectory }\end{array}$ & $\begin{array}{c}\text { Phase } \\
\text { Variations }\end{array}$ & Free? \\
\hline DTW [6] & $\checkmark$ & & & & $\checkmark$ \\
\hline LCSS [3] & $\checkmark$ & & & & \\
\hline ERP [4] & $\checkmark$ & & & & \\
\hline EDR [5] & $\checkmark$ & & & & \\
\hline DISSIM [7] & & $\checkmark$ & & & $\checkmark$ \\
\hline MA [8] & & & & $\checkmark$ & \\
\hline EDwP & $\checkmark$ & $\checkmark$ & $\checkmark$ & $\checkmark$ & $\checkmark$ \\
\hline
\end{tabular}

\section{PROBlem Formulation}

Definition 1: TRAJECTORY: A trajectory $T=$ $\left\{s_{1}, \cdots, s_{n}\right\}$ is a temporally ordered sequence of spatiotemporal points (st-points). An st-point $s=\left(\left[v_{1}, \cdots, v_{d}\right], t\right)$ contains a d-dimensional feature vector describing the spatial attributes and a timestamp $t$ encoding the time at which the location is recorded.

For simplicity, we use trajectories embedded in a 2D plane. The first two dimensions in each st-point denotes the spatial co-ordinates and the third dimension denotes the timestamp.

Definition 2: SUB-TRAJECTORY: $T_{1}$ is a sub-trajectory of $T_{2}$ if $\forall i, 1 \leq i \leq\left|T_{1}\right| T_{1} . s_{i}=T_{2} . s_{a+i}$, where $\exists a, 0 \leq a \leq$ $\left(\left|T_{2}\right|-\left|T_{1}\right|\right)$. The relationship is denoted using $T_{1} \subseteq T_{2}$.

To denote the specific sequence of st-points that define a sub-trajectory $T_{1}$ of $T_{2}$, we use the notation $T_{1}=T_{2}[a, . ., b]$.

Definition 3: SPATIO-TEMPORAL SEgMENT: A spatiotemporal segment $e=\left[s_{1}, s_{2}, f(\cdot)\right]$ represents a segment connecting two temporally consecutive st-points $s_{1}$ and $s_{2}$ through an interpolating function $f(\cdot) . f(\cdot)$ models the movement of the object in the intermediate time interval $s_{1 t}$ to $s_{2 t}$.

We use $e . s_{1}$ and $e . s_{2}$ to denote the two endpoints of $e$ respectively. $s \in e$ denotes an st-point $s$ lying within stsegment $e$. Spatio-temporal segments (st-segments) are better descriptors of trajectories, since they characterize the entire trajectory shape under a given set of observations. We assume $f(\cdot)$ to be a straight line connecting $s_{1}$ and $s_{2}$ and thus represent an st-segment as $e=\left[s_{1}, s_{2}\right]$. Certainly, more accurate functions can be used to interpolate points in the presence of additional information such as the underlying road network [15]. We choose linear since it approximates movements well [7], [16], [17].

With the introduction of an st-segment, hereon, a trajectory is represented as a sequence of segments rather than points. The notion of a sub-trajectory is extended analogously. The length of a trajectory $T$ is defined as follows:

$$
\operatorname{length}(T)=\sum_{\forall e_{i} \in T} \text { length }\left(e_{i}\right)
$$

where length $\left(e_{i}\right)=\operatorname{dist}\left(e_{i} . s_{1}, e_{i} . s_{2}\right)$. The speed within $e$ is defined as $\operatorname{speed}(e)=\frac{\text { length }(e)}{\text { e.s } s_{t}-e \cdot s_{1}}$.

\section{A. Edit Distance with Projections (EDwP)}

Conceptually, given two trajectories $T_{1}$ and $T_{2}$ that are represented as segment sequences, EDwP computes the cheapest set of edits that make them identical. EDwP performs two kinds of edits: replacements and inserts.

Replacement: The replacement operation, denoted by $r e p\left(e_{1}, e_{2}\right)$, represents the operation where the segment $e_{1}$ is matched with $e_{2}$. This match adds a cost, defined as follows:

$$
\operatorname{rep}\left(e_{1}, e_{2}\right)=\operatorname{dist}\left(e_{1} . s_{1}, e_{2} . s_{1}\right)+\operatorname{dist}\left(e_{1} . s_{2}, e_{2} . s_{2}\right)
$$

where $\operatorname{dist}\left(s_{1}, s_{2}\right)$ denotes the euclidean distance between points $s_{1}$ and $s_{2}$. rep $\left(e_{1}, e_{2}\right)$ is symmetric and the cost is proportional to the spatial distance between the endpoints of $e_{1}$ and $e_{2}$. Thus, if $e_{1}$ and $e_{2}$ are identical, the cost would rightly evaluate to zero.

Insert: While $\operatorname{rep}(.,$.$) allows us to match segments, match-$ ing trajectories based only on already sampled points (and such segments) is inadequate due to reasons outlined in Sec. II. The ins $\left(e_{1}, e_{2}\right)$ operation introduces extra points to aid robust matching. In particular, ins $\left(e_{1}, e_{2}\right)$ denotes the operation that inserts a point $p^{\text {ins }\left(e_{1}, e_{2} . s_{2}\right)}$ into $e_{1}$, effectively splitting $e_{1}$ into two segments $\left[e_{1} \cdot s_{1}, p^{i n s\left(e_{1}, e_{2} \cdot s_{2}\right)}\right]$ and $\left[p^{i n s\left(e_{1}, e_{2} \cdot s_{2}\right)}, e_{1} \cdot s_{2}\right]$. The point $p^{i n s\left(e_{1}, e_{2} . s_{2}\right)}$ is determined so that the first segment $\left[e_{1} . s_{1}, p^{i n s\left(e_{1}, e_{2} . s_{2}\right)}\right]$ is best aligned with $e_{2}$; thus, $p^{i n s\left(e_{1}, e_{2} . s_{2}\right)}$ is effectively the point on $e_{1}$ that is spatially closest to $e_{2} . s_{2}$ :

$$
p^{i n s\left(e_{1}, e_{2} \cdot s_{2}\right)}=\underset{p \in e_{1}}{\arg \min } \operatorname{dist}\left(p, e_{2} \cdot s_{2}\right)
$$

Given the construction, we refer to $p^{i n s\left(e_{1}, e_{2} \cdot s_{2}\right)}$ as the projection of $e_{2} . s_{2}$ on to $e_{1}$. The timestamp for the new spatial point is intuitively in proportion with the partition that it induces within $e_{1}$ :

$$
p_{t}^{i n s\left(e_{1}, e_{2} \cdot s_{2}\right)}=e . s_{1 t}+\frac{\operatorname{dist}\left(e . s_{1}, p^{i n s\left(e_{1}, e_{2} \cdot s_{2}\right)}\right)}{\operatorname{speed}\left(e_{1}\right)}
$$

The ins $\left(e_{1}, e_{2}\right)$ operation does not involve any cost; the first part of the split segment of $e_{1}$ is expected to be matched to $e_{2}$ in the next step, wherein the cost would be incurred.

From the perspective of trajectories, the operation $\operatorname{ins}\left(T_{1} . e_{1}, T_{2} . e_{1}\right)$ modifies $T_{1}$ by inducing the split on $T_{1} \cdot e_{1}$ as outlined above. Following this operation, $T_{1}$ has one additional segment, where the first two segments are the partitions of $T_{1} . e_{1}$ from the split followed by segments $T_{1} \cdot e_{2}$ to $T_{1} \cdot e_{\left|T_{1}\right|}$. Hereon, we overload the ins(.,.) notation to use trajectories, so that $\operatorname{ins}\left(T_{1}, T_{2}\right)$ is meant to denote exactly the same split as ins $\left(T_{1} . e_{1}, T_{2} . e_{1}\right)$. Thus, ins $\left(T_{1}, T_{2}\right)$ denotes the insertion defined on the first segments of the trajectories.

Example 1: In Fig. 2(a), the ins $\left(T_{1}, T_{2}\right)$ splits $T_{1} . e_{1}$ by inserting the new point $(0,7,21)$ within $T_{1}$, as illustrated in the figure. Let $T_{1}^{\prime}$ denote the modified version of $T_{1}$ after the insert operation (i.e., the output of ins $\left(T_{1}, T_{2}\right)$ ); the following step would then invoke $\operatorname{rep}\left(T_{1}^{\prime} \cdot e_{1}, T_{2} . e_{1}\right)$ so that the segments $[(0,0,0),(0,7,21)]$ and $[(2,0,0),(2,7,14)]$ be matched incurring the cost:

$\operatorname{dist}((0,0,0),(2,0,0))+\operatorname{dist}((0,7,21),(2,7,14))=2+2=4$

With the formalization of the edit operations, we next define the proposed distance function EDwP between trajectories 

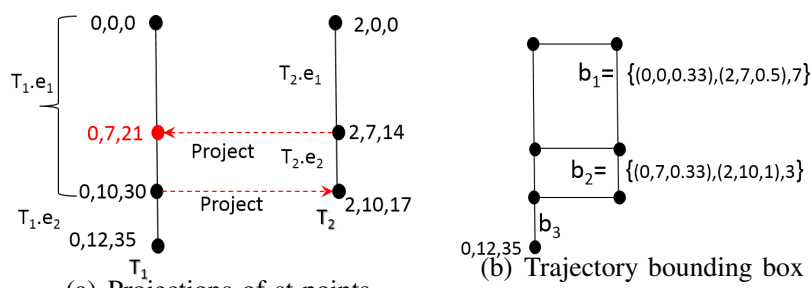

(a) Projections of st-points

Fig. 2. (a). Illustrates the idea of projections. (b). The trajectory bounding box constructed over $T_{1}$ and $T_{2}$ in Fig. 2(a). The tBoxSeq label for $b_{3}$ is omitted since it contains just one segment.

$T_{1}$ and $T_{2}$.

$$
\begin{aligned}
& \operatorname{EDwP}\left(T_{1}, T_{2}\right)= \\
& \left\{\begin{array}{cc}
0 & \text { if }\left|T_{1}\right|=\left|T_{2}\right|=0 \\
\infty & \text { else if }\left|T_{1}\right|=0 \text { or }\left|T_{2}\right|=0 \\
\min \left\{\operatorname{EDwP}\left(\operatorname{Rest}\left(T_{1}\right), \operatorname{Rest}\left(T_{2}\right)\right)+\quad\right. \text { otherwise }
\end{array}\right.
\end{aligned}
$$

where $\operatorname{Rest}(T)$ is the sub-trajectory $T[2, \cdots,|T|]$ containing all segments except T.e $e_{1}$. Coverage quantifies the importance of an edit based on how representative the segments being edited are of the overall trajectories.

$$
\text { Coverage }\left(e_{1}, e_{2}\right)=\text { length }\left(e_{1}\right)+\text { length }\left(e_{2}\right)
$$

Thus, larger segments have more weight on the overall distance than smaller segments.

Example 2: The cheapest sequence of edits to convert $T_{1}$ to $\mathrm{T}_{2}$ in Fig. 2(a) is illustrated in Fig. 3 where the different steps are represented in the sub-figures. Adding the cost of each edit, $\operatorname{EDwP}\left(T_{1}, T_{2}\right)=89.65$.

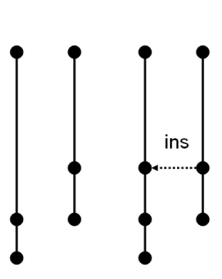

(a)

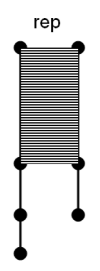

(c)

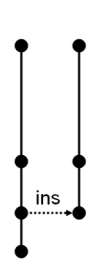

(d)

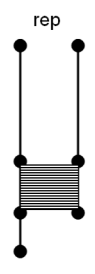

(e)

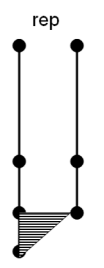

(f)
Fig. 3. Illustration of EDwP while matching the trajectories in Fig. 2(a).

Since EDwP computes the cumulative sum of each edit, in most cases, the distance is likely to increase monotonically with the length of the trajectories (unless the extra length improves the alignment, as could happen in certain cases). In certain situations, the average distance between matched points is more desirable than the cumulative distance. For such cases, we length normalize EDwP.

$$
\operatorname{EDwP}_{\text {avg }}\left(T_{1}, T_{2}\right)=\frac{\operatorname{EDwP}\left(T_{1}, T_{2}\right)}{\text { length }\left(T_{1}\right)+\text { length }\left(T_{2}\right)}
$$

Table II summarizes the novel features of EDwP that enables it to overcome all of the weaknesses of existing techniques without compromising on any of their positive aspects. At
TABLE II. FEATURES OF EDWP THAT SOLVE ISSUES OUTLINED IN TABLE I.

\begin{tabular}{|l|c|c|c|c|}
\hline Local & \multicolumn{3}{|c|}{ Sampling Rate Variations } & Threshold \\
Time & \multicolumn{1}{|c|}{$\begin{array}{l}\text { Inter- } \\
\text { Shifts }\end{array}$} & $\begin{array}{c}\text { Intra- } \\
\text { trajectory }\end{array}$ & $\begin{array}{c}\text { Phase } \\
\text { trajectory }\end{array}$ & Variations \\
\hline $\begin{array}{l}\text { Dynamic Pro- } \\
\text { gramming }\end{array}$ & Projections & Coverage & Segment alignment & $\begin{array}{l}\text { Parameter- } \\
\text { free }\end{array}$ \\
\hline
\end{tabular}

the same time, similar to the quadratic computation costs of DTW, LCSS, ERP, and EDR, the cost of $\operatorname{EDwP}\left(T_{1}, T_{2}\right)$ is $O\left(\left(\left|T_{1}\right|+\left|T_{2}\right|\right)^{2}\right)$

Example 3: To establish how EDwP improves on the deficiencies of existing techniques, let us revisit Figs. 1(b) and $1(c)$. Here, $\operatorname{EDwP}\left(T_{1}, T_{2}\right)$ corresponding to Fig. 1(b) and Fig. $1(c)$ are 20591.26 and 582 respectively. Compared to EDR, EDwP correctly identifies that the trajectories in Fig. 1(c) are significantly more similar than those in Fig. 1(b).

\section{INDEX STRUCTURE}

Due to the quadratic computation cost of EDwP, performing sequential scans across entire databases is not scalable. Thus, we develop an index structure called TrajTree to answer $k$-NN queries efficiently on large trajectory databases.

Like LCSS, DTW and EDR, EDwP is non-metric due to violating triangular inequality.

Theorem 1: EDwP does not satisfy triangular inequality.

Proof: See Sec. A in Appendix.

Due to Theorem 1, generic indexing techniques that are reliant on triangular inequality based pruning cannot be applied. Thus, we formulate the concept of bounding boxes for trajectories and organize the search space in a hierarchical fashion.

\section{A. Indexing trajectories using bounding boxes}

Generally, a bounding box, such as in R-trees [11], summarizes a set of objects such that given a query, its distance to the bounding box is lower than the distances to all of the constituent objects within the box. Generalizing this idea to trajectories, however, is not straightforward. A number of questions arise.

- How do you construct a tight bounding box on a set of trajectories? The most intuitive approach is to describe a set of trajectories as a sequence of bounding boxes. An example is shown in Fig. 2(b). While the bounding box for trajectories in Fig. 2(a) is straight-forward, the scenario in Fig. 4(a) is much more complex. As can be seen, to describe all three trajectories using a sequence of tight bounding boxes, we need to partition each trajectory into a large number of segments. Otherwise, the tightness is compromised. Generating the optimal partitioning scheme is difficult, since they are not based on any global alignment. Rather, the analysis needs to happen in the subtrajectory space, where the optimal partitioning scheme allows us to identify sub-trajectories that are in close spatial proximity and thereby, allowing summarization through a sequence of tight bounding boxes.

- Given a set of bounding boxes that have already been built, where should a new trajectory be inserted? Ideally, 


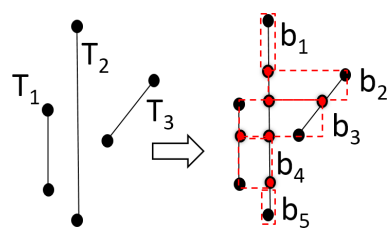

(a)

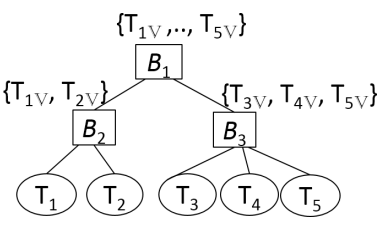

(b)
Fig. 4. (a) The ideal bounding box on the shown three trajectories. Red points denote projected points and the red dashed boxes denote the bounding boxes. (b) Demonstrates the structure of TrajTree. The non-leaf square nodes represent tBoxSeqs, and the circular leaf nodes represent trajectories. $T_{i_{\mathbb{V}}}$ represents the vantage descriptors stored at each node.

it should be inserted on the bounding box that undergoes minimum expansion in volume.

- How can bounding boxes be used to compute a lower bound? In the space of high-dimensional points, this operation is trivial since comparing to the corners of the box is enough. The same strategy does not transfer in the space of trajectories. Additionally, computing the EDwP between a query trajectory and a bounding box does not provide a lower bound.

Existing literature do not answer the above questions, which necessitates a deeper analysis for a formal treatment of the above questions. We begin this analysis by introducing the following definitions.

Definition 4: SPATIO-TEMPORAL BOX: A spatiotemporal box (st-box) $b=\left(s_{1}, s_{2}\right.$, minL) is a bounding box constructed over a set of st-segments. The st-points $s_{1}$ and $s_{2}$ represent the spatial coordinates of the diagonals of b. minL denotes the minimum length of all segments enclosed in $b$.

We use the notations $s \in b$, and $e \in b$ to denote that an st-point $s$ or st-segment $e$ is bounded within $b$.

Definition 5: TRAJECTORY BOX SEQUENCE: A trajectory box sequence (tBoxSeq) is a sequence of st-boxes. The operation of constructing a tBoxSeq over a set of trajectories $\mathbb{T}=\left\{T_{1}, \ldots, T_{n}\right\}$ is denoted as $\mathcal{B}=t \operatorname{BoxSeq}(\mathbb{T})$. The volume $\operatorname{Vol}(\mathcal{B})$ of $\mathcal{B}$ is $\sum_{i}^{|\mathcal{B}|} \operatorname{Vol}\left(\mathcal{B} . b_{i}\right)$. In $2 D, \operatorname{Vol}\left(\mathcal{B} . b_{i}\right)$ is simply the area of the bounding box $\mathcal{B} . b_{i}$.

An example of a tBoxSeq is shown in Fig. 2(b). Next, we extend the existing definitions and notations to tBoxSeqs.

- Sub-trajectory: Trajectory $T \subseteq \mathcal{B}$, if $\forall i, 1 \leq i \leq$ $|T|, T . e_{i} \in \mathcal{B} . b_{a+i}$ for some $0 \leq a \leq(|\mathcal{B}|-|\bar{T}|)$.

- $\quad \operatorname{dist}(\mathbf{s}, \mathbf{b}):$ The distance between an st-point $s$ and an st-box $b$ is $\min _{\forall p \in b}\{\operatorname{dist}(s, p)\}$.

Projection: The projection of a point $s$ on an st-box $b$ is defined as $p^{i n s(b, s)}=\arg \min _{p \in b} \operatorname{dist}(s, p)$. The reverse projection $p^{i n s(e, b)}=\arg \min _{p \in b} \operatorname{dist}(s, p) \forall s \in$ $e$ of $b$ on a segment $e$ is defined analogously.

- $\quad$ Replace and Insert: By incorporating the generalized formulations of projection and $\operatorname{dist}(\cdot, \cdot)$, replace and insert edits are computed accordingly.

- Coverage: Coverage $($ T.e, $\mathcal{B} . b)=$ length $(e)+$ length (b.min $L)$, where $T$ is a trajectory and $\mathcal{B}$ is a tBoxSeq.

All existing notations such as $\operatorname{Rest}(\mathcal{B}), \mathcal{B}[m, \cdots, n]$, etc. are extended analogously.

\section{B. Constructing tBoxSeqs}

To construct tBoxSeqs, the optimal partitioning scheme needs to be computed in the sub-trajectory space. Towards that goal, we define a sub-trajectory distance function, $\mathrm{EDwP}_{\text {sub }}$. The goal in $\operatorname{EDwP}_{\text {sub }}\left(T_{1}, T_{2}\right)$ is to identify the sub-trajectory in $T_{2}$ that is most similar to $T_{1}$. As in the global alignment using EDwP, EDwP $\mathrm{E}_{\text {sub }}$ uses the same edit operations of replace and insert to partition and align trajectories. However, the edits are used in a different manner to identify the most similar subtrajectory rather than the optimal global alignment. Using the generalized formulations above, the arguments in EDwP can either be a trajectory or tBoxSeq. Formally, $\mathrm{EDwP}_{\text {sub }}$ is defined as follows.

$$
\begin{aligned}
& \text { PrefixDist }(T, \mathcal{B})= \\
& \left\{\begin{array}{lr}
0 & \text { if }|T|=0 \\
\infty & \text { if }|\mathcal{B}|=0 \\
\min \{\operatorname{PrefixDist}(\operatorname{Rest}(T), \operatorname{Rest}(\mathcal{B}))+\quad \text { otherwise } & \left(\text { rep }\left(T . e_{1}, \mathcal{B} . b_{1}\right) \times \operatorname{Coverage}\left(T . e_{1}, \mathcal{B} . b_{1}\right),\right) \\
& \operatorname{PrefixDist}(\operatorname{ins}(T, \mathcal{B}), \mathcal{B}) \\
& \operatorname{PrefixDist}(T, \operatorname{ins}(\mathcal{B}, T))\}
\end{array}\right.
\end{aligned}
$$$$
\operatorname{EDwP}_{\text {sub }}(T, \mathcal{B})=\min _{1 \leq i \leq|\mathcal{B}|}\{\operatorname{PrefixDist}(T, \mathcal{B}[i, . .,|\mathcal{B}|])\}
$$

where $T$ is a trajectory and $\mathcal{B}$ is a tBoxSeq. Note that the only differences between EDwP and PrefixDist are the initialization conditions for $|T|=0$ and $|\mathcal{B}|=0$. This modification allows PrefixDist to skip suffixes of $\mathcal{B}$ without incurring any penalty. Consequently, PrefixDist computes the prefix of $\mathcal{B}$ that best matches to $T$. Since our goal is to compute the best-matching sub-trajectory (or a sub-sequence of a tBoxSeq), along with skipping suffixes, we should also be able to skip prefixes. Skipping prefixes is achieved by the $\min \{\ldots\}$ condition in the $\operatorname{EDwP}_{\text {sub }}(T, \mathcal{B})$ equation; $\operatorname{EDwP}_{\text {sub }}(T, \mathcal{B})$ explicitly considers suffixes of $\mathcal{B}$ as candidates for $T$ to match with using PrefixDist. Thus, overall, the proposed systematic combination allows for skipping any prefix as well as any suffix of $\mathcal{B}$, which allows the possibility of aligning $T$ with any contiguous sub-sequence of $\mathcal{B}$. Clearly, $\operatorname{EDwP}_{\text {sub }}(T, \mathcal{B})$ is asymmetric. The computation cost of $\mathrm{EDwP}_{\text {sub }}$ is same as EDwP, which is $O\left((|T|+|\mathcal{B}|)^{2}\right)$. Even though PrefixDist needs to be computed for each suffix of $\mathcal{B}$, by employing dynamic programming, the results for a suffix $S$ can be reused while computing the distance for a larger suffix $S^{\prime} \supseteq S$.

Equipped with $\mathrm{EDwP}_{s u b}$, to compute the tBoxSeq on two trajectories, first, the optimal subsequence alignment is identified, and then an st-box is computed corresponding to each replace operation. To generalize the operation over a set of trajectories $\mathbb{T}=\left\{T_{1}, \cdots, T_{|\mathbb{T}|}\right\}$, the following iterative procedure is followed.

1) Initialize tBoxSeq, $\mathcal{B}=$ createT Box $\operatorname{Seq}\left(T_{1}\right)$

2) $\forall i, 2 \leq i \leq|\mathbb{T}|$

$$
\text { a) } \mathcal{B}=\text { createT Box } \operatorname{Seq}\left(T_{i}, \mathcal{B}\right)
$$

where the createTBoxSeq $\left(T_{i}, \mathcal{B}\right)$ represents a tBoxSeq based on the $\operatorname{EDwP}_{\text {sub }}\left(T_{i}, \mathcal{B}\right)$ alignment, and creating an stbox for each aligned segment. The final $\mathcal{B}$ is the desired tBoxSeq over all trajectories in $\mathbb{T}$. 
Example 4: Revisiting Fig. 2(a), $E D w P_{\text {sub }}\left(T_{1}, T_{2}\right)=$ 89.65 and $E D w P_{\text {sub }}\left(T_{2}, T_{1}\right)=80$. Fig. 2(b) demonstrates the tBoxSeq corresponding to the trajectories in Fig. 2(a). Although EDwP $P_{\text {sub }}$ is not symmetric, the tBoxSeq constructed is identical in this example regardless of the order. For $\operatorname{EDwP} P_{\text {sub }}\left(T_{2}, T_{1}\right)$, first ins $\left(T_{2} . e_{1}, T_{1} . e_{1}\right)$ inserts an additional point in $T_{1}$. Let us denote this partitioned $T_{1}$ as $T_{1}^{\prime}$. Following this, $\operatorname{rep}\left(T_{1}^{\prime} \cdot e_{1}, T_{2} . e_{1}\right)$ and $\operatorname{rep}\left(T_{1}^{\prime} \cdot e_{2}, T_{2} . e_{2}\right)$ are the two edits. $T_{1} \cdot e_{2}$ is left unmatched.

\section{Computing lower bound from tBoxSeq}

For tBoxSeqs to be useful in pruning the search space, we need to show that a lower bound on the distances to the constituent trajectories can be derived. It is easy to see that $\operatorname{EDwP}(T, \mathcal{B}) \leq \operatorname{EDw} P\left(T, T_{i}\right) \forall T_{i} \in \mathcal{B}$, where $\mathcal{B}$ is a tBoxSeq. This follows from the fact that EDwP computes a global alignment. Thus, we focus on $\mathrm{EDwP}_{\text {sub }}$ and re-use it for computing a lower bound as well. Specifically, we prove the following theorem.

Theorem 2: Given a set of trajectories $\mathbb{T}=\left\{T_{1}, . ., T_{n}\right\}$ and a query trajectory $Q$, let $\mathcal{B}=t \operatorname{Box} S e q(\mathbb{T})$. We claim $\operatorname{EDwP}_{\text {sub }}(Q, \mathcal{B}) \leq E D w P(Q, T) \forall T \in \mathbb{T}$.

PROOF: We first establish the following lemma.

Lemma 1: Let $T_{s} \subseteq T$ be two trajectories where $T_{s}=$ $T[1, \ldots, n], 1 \leq n \leq|T|$, In other words, $T_{s}$ is a prefix of $T$. Then, for any trajectory $Q$,

$$
\operatorname{PrefixDist}(Q, T) \leq \operatorname{EDwP}\left(Q, T_{s}\right) \forall T_{s}
$$

\section{PROOF BY INDUCTION:}

Base Case: Consider, $|T|-\left|T_{s}\right|=0$, i.e., $T_{s}=T$

It is easy to see that $\operatorname{PrefixDist}(Q, T) \leq \operatorname{EDwP}\left(Q, T_{s}\right)$ since all computations in $\operatorname{PrefixDist}(Q, T)$, and $\operatorname{EDwP}\left(Q, T_{s}\right)$ are identical except when $Q=\emptyset$ and $T_{s}=T \neq \emptyset$. In this case, PrefixDist $(Q, T)=0$ and $\operatorname{EDwP}\left(Q, T_{s}\right)=\infty$. Thus, PrefixDist $(Q, T) \leq \operatorname{EDwP}\left(Q, T_{s}\right)$.

Induction Step: Assume, $\forall Q, \quad \operatorname{PrefixDist}(Q, T) \leq$ $\operatorname{EDwP}\left(Q, T_{s}\right)$, where $|T|-\left|T_{s}\right|=k$. We need to now show that PrefixDist $(Q, T) \leq \operatorname{EDwP}\left(Q, T_{s}\right)$ holds for $|T|-\left|T_{s}\right|=k+1$. Let,

$$
T_{s s} \subseteq T \text { be } T_{s s}=T[1, . .,(|T|-1)]
$$

Thus,

$$
\left|T_{s s}\right|-\left|T_{s}\right|=k
$$

Now, let $\mathbb{E}$ be the optimal set of edit operations for PrefixDist $\left(Q, T_{s s}\right)$. From induction hypotheses,

$$
\operatorname{PrefixDist}\left(Q, T_{s s}\right) \leq \operatorname{EDwP}\left(Q, T_{s}\right)
$$

Among all sequences of edits that will be explored in PrefixDist $(Q, T)$, one of them will involve $\mathbb{E}$ followed by the optimal edits for PrefixDist $(\emptyset, T[|T|, . .,|T|])=0$. This follows from the fact that to optimally align $T$, its immediate sub-trajectory $T_{s s}$ needs to be aligned first, followed by the optimal alignment for the remaining portions. Thus, there exists a sequence of edits where

$$
\operatorname{PrefixDist}(Q, T) \leq \operatorname{EDwP}\left(Q, T_{s}\right)
$$

Lemma 2: For any two trajectories $T_{1}$ and $T_{2}$, $\operatorname{EDwP}_{\text {sub }}\left(T_{1}, T_{2}\right) \leq \operatorname{EDwP}\left(T_{1}, T_{s}\right) \forall T_{s} \subseteq T_{2}$.
Proof By CONTRADICTION: Assume there is a sub-trajectory $T_{s}=T_{2}[a, . ., b]$ such that $\operatorname{EDwP}_{s u b}\left(T_{1}, T_{2}\right)>\operatorname{EDwP}\left(T_{1}, T_{s}\right)$. Thus, from the definition of PrefixDist,

$$
\exists T_{s}^{\prime} \supseteq T_{s}, \operatorname{PrefixDist}\left(T_{1}, T_{s}^{\prime}\right)>\operatorname{EDwP}\left(T_{1}, T_{s}\right)
$$

where $T_{s}^{\prime}=T_{2}\left[a, \ldots,\left|T_{2}\right|\right]$. However, using Lemma 1, this result contradicts the base assumption. Thus, proved.

It is easy to see that Lemma 2 also extends to tBoxSeqs.

Corollary 1: For any trajectory $T$ and $t$ BoxSeq $\mathcal{B}$, $\operatorname{EDwP}_{\text {sub }}(T, \mathcal{B}) \leq \operatorname{EDwP}\left(T, T_{s}\right) \forall T_{s} \subseteq \mathcal{B}$.

Corollary 1, however, is not enough to prove Theorem 2. More specifically, $t \operatorname{Box} \operatorname{Seq}(\mathbb{T})$ does not guarantee that $\forall T \in$ $\mathbb{T}, T \subseteq t \operatorname{Box} \operatorname{Seq}(\mathbb{T})$. To illustrate the issue, consider Figs. 2(a) and 2(b). As can be seen, $T_{1} \nsubseteq \mathcal{B}$ since $T_{1} . e_{1} \notin \mathcal{B} . b_{1}$. Although the shape of $T_{1}$ has been captured in $\mathcal{B}, \mathcal{B}$ divides $T_{1}$ into a higher number of partitions. Thus, we next proceed towards proving the following Lemma.

Lemma 3: Consider two trajectories $T_{1}$ and $T_{2}$ such that both contain one st-segment each. If a new trajectory $T_{2}^{\prime}=$ $\left[\left[T_{2} . e_{1} . s_{1}, p\right],\left[p, T_{2} . e_{1} . s_{2}\right]\right]$ is created by inserting an additional point $p$ on $T_{2} . e_{1}$, then $\operatorname{EDwP}\left(T_{1}, T_{2}^{\prime}\right) \leq \operatorname{EDwP}\left(T_{1}, T_{2}\right)$.

Proof: See Sec. B in Appendix.

Lemma 3 can easily be extended to the following corollary.

Corollary 2: Given three trajectories $T_{1}, T_{2}$ and $T_{2}^{\prime}$, where $T_{2}^{\prime}$ is generated by inserting any arbitrary number of points in arbitrary st-segments $e \in T_{2}, \operatorname{EDwP}\left(T_{1}, T_{2}^{\prime}\right) \leq \operatorname{EDwP}\left(T_{1}, T_{2}\right)$.

By combining Corollaries 1 and 2, Theorem 2 is proved.

\section{Building the index structure}

The structure of TrajTree is similar to existing tree-based spatial access methods such as R-trees [11]. More specifically, a tree is built, where the root represents the tBoxSeq over the entire database. Next, trajectories in the root are partitioned into $b f$ different groups, and the tBoxSeq on each group forms a child node of the root. The process is repeated recursively till a node is reached that contains less than $n$ trajectories. Thus, each non-leaf node represents a tBoxSeq, and leaves correspond to trajectories. The structure of the tree is shown in Fig. 4(b). There are two parameters: the branching factor $b f$ and the minimum size of each group $n$. In this paper, we focus on an in-memory setting and $n$ does not have a significant impact on the performance. For an on-disk implementation, existing techniques based on the disk page size can be used to optimize $n$. $b f$, on the other hand, drastically impacts the performance of the index structure. If $b f$ is set too low, the lower bounds computed using Theorem 2 would be loose. On the other hand, for a large $b f$, the lower bounds would be tight, but more computations would be performed at each level of the tree. It is therefore critical to obtain the right balance between the branching factor and the tightness of the lower bounds. Towards that goal, we use Alg. 1 to partition trajectories at each node.

We build a subset of trajectories $\mathbb{P} \subset \mathbb{D}$, where $\mathbb{P}$ comprises of a set of diverse trajectories. $\mathbb{P}$ is initialized to a random trajectory from $\mathbb{D}$ (line 3 ). Next, we add trajectories to $\mathbb{P}$ by choosing the database trajectory that is most diverse from all trajectories in $\mathbb{P}$ (the max of min construction in line 5). The 


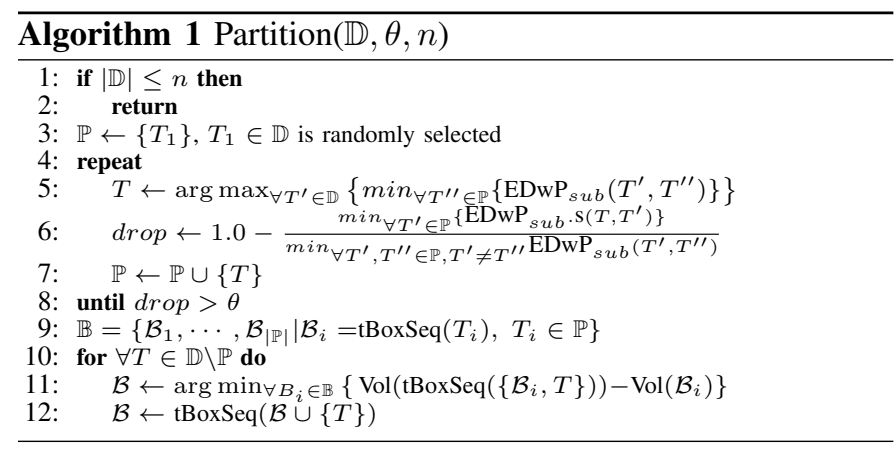

diversity within $\mathbb{P}$ is quantified using the minimum distance between any pair of its constituent trajectories (denominator in RHS of line 6), and we keep expanding $\mathbb{P}$ as long as the marginal fractional drop in its diversity (drop in line 6) is under a threshold $\theta$. Each trajectory in $\mathbb{P}$ now acts as a pivot and is initialized to a tBoxSeq. Hereon, each of the remaining trajectories in $\mathbb{D}$ is added to the tBoxSeq that undergoes the minimum expansion in volume (lines 9-12). Thus, instead of $b f$, we use $\theta$ to dynamically adjust the branching factor based on the properties of the dataset.

\section{E. Vantage Points}

In this section, we further boost the pruning power of TrajTree by distributing a set of vantage points (VP). By consolidating the unique viewpoints of the VPs, a vantage descriptor is generated for each trajectory, which is then used to compute an upper bound on the maximum possible distance between the query and any trajectory in the $k$-NN answer set. The upper bound is then used in a manner similar to best-first search to prune the search space. The idea of VPs is inspired from Lipschitz embedding [10].

Definition 6: VAnTAge PoInt: A VP is a spatial point in the trajectory space. The distance between a trajectory $T$ and a VP $v$ is the distance between $v$ and the point $p$ in $T$ that is closest to $v$. Note, $p$ may not necessarily be a sampled point.

$$
\left.\operatorname{VP}-\operatorname{dist}(T, v)=\min _{\forall e \in T}\{\underset{p \in e}{\arg \min } \operatorname{dist}(v, p))\right\}
$$

where $e$ is an st-segment in $T$.

Definition 7: VANTAGE

DESCRIPTOR:

Given $a$ set of VPs $\mathbb{V}=\left\{v_{1}, \cdots, v_{d}\right\}$, the vantage descriptor of $T$ is a d-dimensional feature vector $T_{\mathbb{V}}=\left[V P-\operatorname{dist}\left(T, v_{1}\right), \cdots, V P-\operatorname{dist}\left(T, v_{d}\right)\right]$. We use $T_{\mathbb{V}}[i]$ to denote the $i_{\text {th }}$ dimension in $T_{\mathbb{V}}$.

In essence, the vantage descriptor of a trajectory captures a feature space representation based on the viewpoints of VPs in $\mathbb{V}$. Next, we define vantage distance between two trajectories.

Definition 8: VANTAGE DistANCE: The vantage distance between trajectories $T_{1}$ and $T_{2}$ is defined as the following:

$$
V D\left(T_{1}, T_{2}\right)=\frac{\sum_{i=0}^{|\mathbb{V}|} 1-\frac{\min \left\{T_{1_{\mathbb{V}}}[i], T_{2_{\mathbb{V}}}[i]\right\}}{\max \left\{T_{1_{\mathbb{V}}}[i], T_{2_{\mathbb{V}}}[i]\right\}}}{|\mathbb{V}|}
$$

Vantage distance builds on the intuition that if trajectories $T_{1}$ and $T_{2}$ are equidistant from most of the distributed VPs, then they are likely to have traveled through similar regions. In other words, $\operatorname{EDwP}\left(T_{1}, T_{2}\right)$ and $V D\left(T_{1}, T_{2}\right)$ are likely to be correlated. Now, let $\mathcal{A}$ be the true $k$-NN answer set with respect to a given query trajectory $Q$, and $\mathcal{A}_{\mathcal{V D}}$ be the $k$-NN based on VPs. We define an upper bound $U B$ as the following

$$
U B=\arg \max _{\forall T^{\prime} \in \mathcal{A}_{\mathcal{V} \mathcal{D}}} \operatorname{EDwP}\left(T^{\prime}, Q\right)
$$

It is easy to see that $\forall T \in \mathcal{A}, \operatorname{EDwP}(T, Q) \leq U B$. Indeed, an upper bound can be computed from any random selection of $k$ trajectories from the database. However, the tightness of the upper bound depends on how well the selected trajectories overlap with $\mathcal{A}$. VPs allow us to make a more informed selection of $k$ database trajectories and thereby, producing a tight upper bound. Additionally, since $V D\left(T_{1}, T_{2}\right)$ operates in the feature space and the computation cost is linear, $V D\left(T_{1}, T_{2}\right)$ is much faster than $\operatorname{EDwP}\left(T_{1}, T_{2}\right)$. To take advantage of these properties, we integrate VPs with TrajTree.

While constructing the index, in addition to computing the tBoxSeq at each node $n, d$ VPs are also distributed and the resultant vantage descriptors of all trajectories in the subtree rooted at $n$ are stored (Fig. 4(b)). The VPs are chosen using the same mechanism used for selecting pivots while splitting a node. Based on the chosen VPs, the vantage descriptor for each trajectory in the subtree of $n$ is stored. Note that the density of VPs increases as lower levels of the tree are reached since the number of trajectories under a subtree decreases with the level. Consequently, resultant vantage descriptors get more refined as the depth of a node in the tree increases.

\section{F. Indexing costs and updates}

Storage Cost: The storage cost depends on the branching factor, which is controlled by $\theta$. Assuming a balanced branching factor of $b f$, the height of the tree is $\log _{b f}|\mathbb{D}|+1$, where $\mathbb{D}$ is the trajectory database. The number of nodes at each level of the tree follows a geometric progression, and is thus bounded by $O\left(\frac{b f|\mathbb{D}|-1}{b f-1}\right)$. Additionally, at each level of the tree, $|\mathbb{D}|$ vantage descriptors are stored. Therefore, total storage is $O\left(\frac{b f|\mathbb{D}|-1}{b f-1}+|\mathbb{V}||\mathbb{D}| \log _{b f}|\mathbb{D}|\right)$, where $|\mathbb{V}|$ is the number of VPs at each node.

Index Construction Cost: Except at the last level of the tree, a cost of $\mathrm{O}(p|\mathbb{D}|)$ is incurred at each level to select the pivots, where $p$ is the number of pivots. The number of pivots at any level $l$ of the tree follows the equation $b f^{l}$. Thus, the total index construction cost is bounded by $O\left(\frac{|\mathbb{D}|^{2}}{b f}\right)$.

Updates: The index construction algorithm bulk-loads all trajectories in the database. To insert a new trajectory, the algorithm remains identical except for omitting the initial step of selecting pivots at each node since existing pivot points are reused. For deleting a trajectory, the corresponding leaf node and the vantage descriptors at all nodes from the leaf to the root are deleted. The tBoxSeqs remain unchanged. As in most hierarchical index structures, updates decrease efficiency since each insert or delete is likely to make the affected tBoxSeqs loose. Thus, to tackle this issue, we track the diversity of the pivots at each node. A node is classified as "poor" if the increase in diversity due to re-computing the pivots is above a threshold. Finally, if the number of "poor" nodes is above a certain ratio, then the index is re-built. 


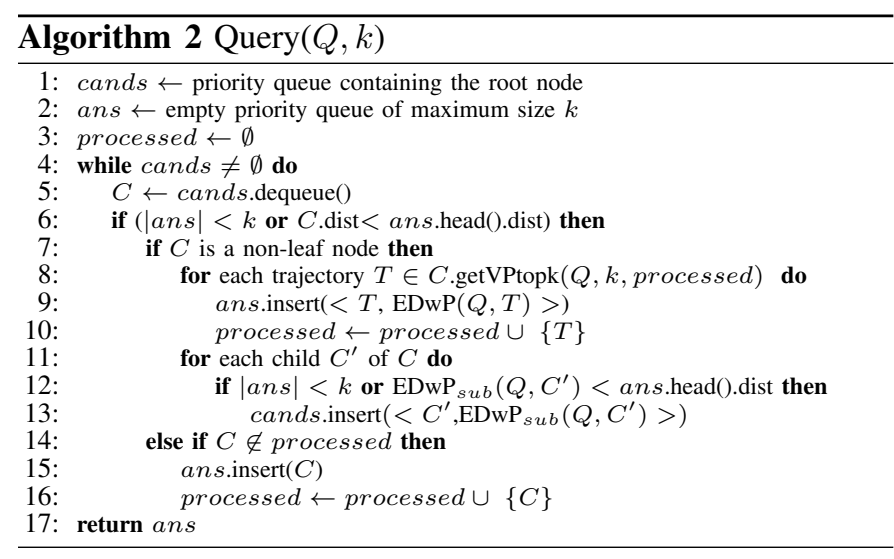

\section{G. Querying Algorithm}

Alg. 2 outlines the pseudocode to answer $k$-NN queries. The algorithm proceeds in a manner similar to best-first search. Two priority queues, cands and ans, are maintained to compute the answer set. cands prioritizes the unexplored nodes in the index based on their distances to the query. Initially, cands contains just the root node. ans maintains the $k$-NN trajectories found at any given state of the searching procedure (line 2). ans is populated in an iterative manner (lines 4-16). At each iteration, the node $C$ with the lowest distance is extracted from cands and processed if it satisfies the constraints (lines 5-6).

Case 1: If $C$ is a tBoxSeq, then the following steps are performed.

1. Update upper bound based on VPs at $C$ : First, the top- $k$ nearest trajectories to $Q$ are computed based on the VPs at $C$. This analysis searches only those trajectories that are under the subtree rooted at $C$. Next, the actual distance to each of the VP-based $k$-NN trajectories are computed to generate an upper bound $U B$ as outlined in Eq. 14 ( $U B$ is ans.head().dist in Alg. 2). Additionally, the trajectories in the VP-based $k$-NN are inserted to ans if they are currently within the top- $k$ (lines $8-10)$. $U B$ is next used to prune the children of $C$.

2. Prune children of $C$ based on the updated lower bound: Once the upper bound from step 1 is derived, $\mathrm{EDwP}_{\text {sub }}$ for each children of $C$ is computed and added to cands if they have a distance below $U B$ (lines 11-13).

Case 2: If $C$ is a trajectory, ans is updated based on $C$ 's distance to query trajectory $Q$ (lines 14-16). As can be seen, a hashset called processed is maintained to track trajectories that have already been evaluated. If not for processed, due to possible overlaps between the VP-based $k$-NN sets of nodes in the same path, trajectories common to both sets would be processed multiple times.

Therefore, to summarize the features of TrajTree querying:

- The $k$-NN answer set is exact and optimal.

- The distances of query trajectory $Q$ to tBoxSeqs prioritize the search order.

- VPs produce a tight upper bound $U B$ by approximating the optimal $k$-NN answer set. Since VPs operate at a feature space, computing $U B$ is fast.

\section{EXPERIMENTS}

In this section, we demonstrate that:

- EDwP is more accurate and robust to noise than stateof-the-art trajectory matching techniques.

- $\quad$ TrajTree is efficient in indexing $k$-NN queries.

\section{A. Experimental Setup}

All our algorithms are implemented in Java 1.6.0 and benchmarked on a PC with 12GB memory and Intel i5 $2.60 \mathrm{GHz}$ quad core processor running Ubuntu 12.10. We use the length normalized EDwP defined in Eq. 4.

Datasets: We use two real trajectory datasets. The first dataset is extracted from the Beijing cab dataset [18]. This dataset contains trajectories of 10,000 cabs tracked over a period of one week in Beijing [18]. Since we would like each trajectory to represent a single trip, we partition a trajectory into two if either the cab is stationary for more than 15 minutes, or the time gap between two consecutive points is more than 15 minutes. Following this procedure, we use a subset 42, 000 trajectories to create our dataset.

Besides vehicular trajectories, we also use the Australian Sign Language (ASL) dataset, which contains trajectories of hand movements that denote 98 different signs such as "alive", "cold", "computer", etc. Thus, each trajectory in the ASL dataset is labeled with the sign it denotes.

Benchmarking Techniques: We compare the accuracy of EDwP with LCSS [3], EDR [5], DISSIM [7] and the semicontinuous assignment model in MA [8]. EDR and LCSS have been shown to outperform DTW [6] and ERP [4] in [5].

Parameters: While EDwP is parameter-free, EDR and LCSS are dependent on a matching threshold. MA has as many as four parameters. We set these parameters as outlined by the respective papers. TrajTree, requires three parameters. The default values of $\theta$ in Alg. 1 and the number of vantage points are set to 0.8 and 80 respectively. The minimum size of a node in TrajTree is set to 10 . The default $k$ in the $k$-NN queries is set to 10 .

\section{B. Accuracy in clean data}

First, we evaluate the accuracy of EDwP in clean data. The ASL dataset contains trajectories from 98 different classes with each class denoting a sign. These trajectories are recorded in controlled environments and are thus clean. To measure accuracy, we perform multi-class classification. We prepare the dataset for the classification experiment by first randomly selecting $c$ classes, and then adding all trajectories under these classes to the classification dataset. Next, using each of the four distance metrics, we perform 10 -fold cross-validation. The class labels of trajectories in the testing set is predicted using nearest neighbor classification. The process is repeated 100 times for consistency.

Fig. 5(a) demonstrates the results as the number of classes is varied from 5 to 25 . EDwP achieves the highest accuracy across all numbers of classes. As the number of classes increases, the classification task gets harder and consequently, the accuracies of all distance metrics decrease. This rate of 


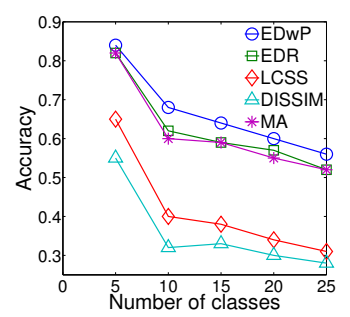

(a)

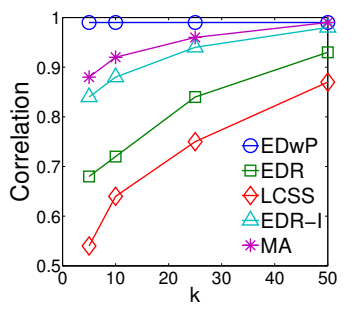

(f) Against $k$

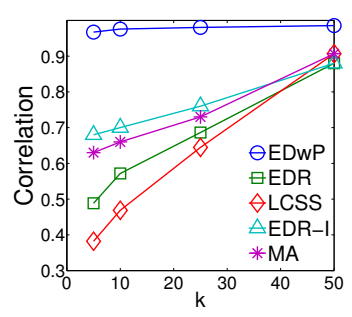

(b) Against $k$

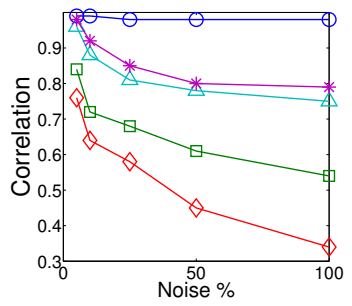

(g) Against $n$

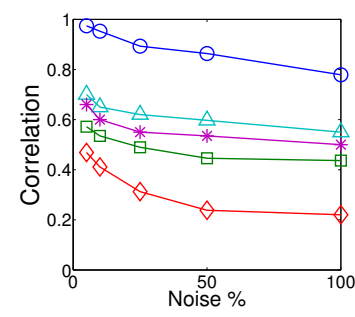

(c) Against $n$

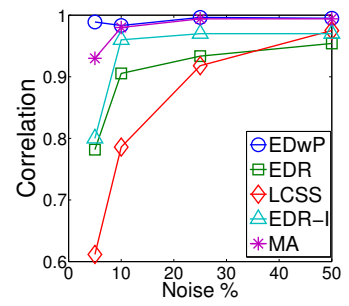

(h) Against $k$

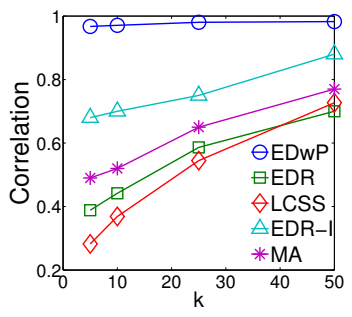

(d) Against $k$

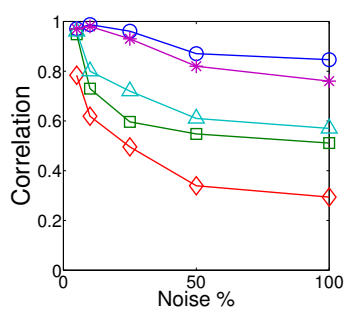

(i) Against $n$

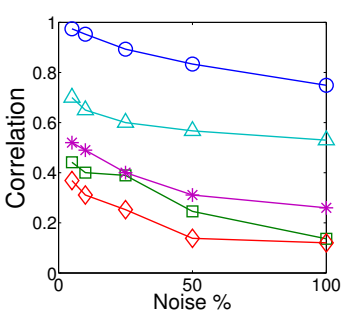

(e) Against $n$

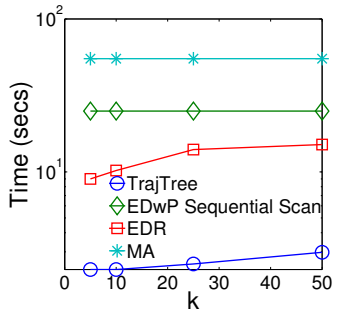

(j) Beijing

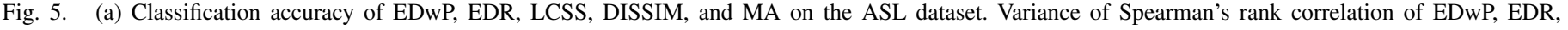

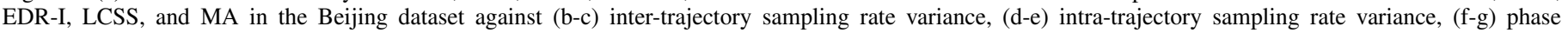

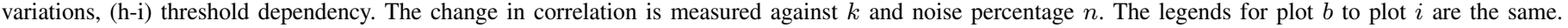
(j) Growth rate of querying time with $k$.

decrease however, is the smallest in EDwP indicating better robustness to matching difficulty. The performance of DISSIM suffers since it is unable to cope with local time shifts.

\section{Accuracy in noisy data}

In this section, we benchmark the robustness of EDwP to sampling noise. We model each of the scenarios discussed in Sec. II and compare the performances of the distance metrics. For all of the experiments in this section, we construct two datasets, $D_{1}$ and $D_{2}$. While $D_{1}$ is clean, the second dataset $D_{2}$ is noisy. The process of injecting noise is dependent on the issue being evaluated. Now, to quantify robustness, we first construct a ground truth result set by computing the $k$-NN list for a randomly chosen query from the clean $D_{1}$ dataset. Next, we re-compute the $k$-NN list for the same query trajectory in the noisy $D_{2}$ dataset. A robust distance metric should adapt to the injected noise and produce an answer set that is close to the $k$-NN on the clean $D_{1}$ dataset. Based on this hypotheses, we compute Spearman's rank correlation coefficient [19] between the two $k$-NN lists. Since the elements in the two $k$-NN lists may not overlap completely, we form a single element set by taking their union. Next, for each element in the union set, we fetch its ranks in $D_{1}$ and $D_{2}$. Finally, we compute the correlation between the two ranked lists. The closer the correlation is to 1 , the more robust the distance metric is.

For a thorough investigation of the performance, we also apply EDR on an interpolated dataset. As discussed in Sec. II, issues related to non-uniform sampling rates can be alleviated by interpolating the dataset with additional points so that all trajectories have a uniform sampling rate. As already discussed, this pre-processing step is extremely expensive and needs to be performed at query time. However, for the purposes of better understanding the impact of sampling rate, we ignore the scalability aspect and perform interpolation on both $D_{1}$ and $D_{2}$ to ensure a uniform sampling rate. Except this preprocessing, the subsequent steps for the $k$-NN query remain identical. We denote EDR on this interpolated scenario as EDR-I. DISSIM is not included in this experiment as it is unable to detect spatial similarity between trajectories moving at dissimilar speeds.

Inter-trajectory sampling variance: In this experiment, $D_{1}$ corresponds to the Beijing dataset. To model variance in inter-trajectory sampling rates in $D_{2}$, without altering the shape of a trajectory $T \in D_{1}$, we randomly select $n \%$ of its segments, and partition them into two by inserting a point. Thus, $D_{2}$ represents the same trajectories in $D_{1}$ at a higher sampling rate. $n$ is the noise parameter that controls the difference in sampling rate between trajectories in $D_{1}$ and $D_{2}$. Fig. 5(b) demonstrates the results at $n=5 \%$ while $k$ is varied between 5 and 50. Fig. 5(c), on the other hand, analyzes the performance at $k=10$ as $n$ is varied to increase the difference in sampling rate between $D_{1}$ and $D_{2}$. Across both $k$ and $n$, EDwP achieves an accuracy that is up to 4 times better than existing techniques. As expected, the correlation increases with $k$ since there is more overlap with the true $k$-NN list as $k$ grows. Regardless of $k$ however, EDwP achieves a rank correlation that is close to 1 due to dynamic interpolation achieved through projections. Against $n$, the performance difference is even more drastic. Even in the worst case scenario, EDwP's correlation with the true answer set is higher than 0.75 . Despite interpolation, EDRI performs worse than EDwP since it is limited to matching only the existing sampled (or interpolated) points. On the other hand, the alignment possibilities for EDwP are infinite due to projections. MA suffers since with the introduction of additional points, the trade off between 'gap points' and matched points change.

Intra-trajectory sampling variance: Similar to the previous experiment, $D_{1}$ corresponds to the Beijing dataset. To model intra-trajectory variance in sampling rates in $D_{2}$, we take only the first half of each trajectory, and within this half, we insert points in $n \%$ of the segments in a manner similar to the previous experiment. Thus, the sampling rate in 
each trajectory in $D_{2}$ is higher in the first half. Figs. 5(d) and 5(e) demonstrate the results. Although the trends are similar to the results for inter-trajectory sampling variance, the performance gap between EDR-I and EDR is significantly higher. Due to the interpolation in the pre-processing step, the difference in sampling rate disappears in EDR-I, and the scenario degenerates to the case of only inter-trajectory sampling rate difference between $D_{1}$ and $D_{2}$. Overall, EDwP is up to $50 \%$ more correlated, since it weights each segment alignment based on its coverage and thus automatically adapts to the variance in the sampling rate.

Phase Variations: To model phase variations, we construct two datasets from Beijing. Specifically, for each trajectory, we randomly choose $n \%$ of its segments and partition each segment into two by inserting an additional point. This altered trajectory is next added to $D_{1}$. Now, the exact same set of segments are partitioned again and added to $D_{2}$. Thus, the sampling rate and the set of altered segments in $D_{1}$ and $D_{2}$ are identical; the only difference lies in the location of the inserted point. As can be seen in Figs. 5(f) and 5(g), although the trends are similar, generally, the performance of EDR and LCSS are better than inter and intra-trajectory sampling variances with MA performing best among existing techniques. Since MA considers aligning a sampled point to a non-sampled point, its performance is stable. EDwP performs the best due to projections and coverage.

Threshold Dependency: Finally, to highlight the impact of threshold dependency, we perturb the locations of $n \%$ of the st-points in each trajectory by a small amount and add it to $D_{2} . D_{1}$ represents the original Beijing dataset. To perturb a point, we draw a circle with the original location as the center and a radius equivalent to the distance traveled in 30 seconds based on the average speed of trajectories in the dataset. The perturbed location is then set to one randomly selected point within the circle. Figs. 5(h) and 5(i) demonstrate the results against $k$ (at $n=10 \%$ ) and $n$ (at $k=10$ ) respectively. While the impact of threshold is not as drastic as with sampling rate variance, all techniques suffer due to relying on thresholds.

\section{Index Performance}

We now verify the efficiency of TrajTree in indexing $k$-NN queries. We compare the querying time of TrajTree with the index structure for EDR [5], and sequential scans over EDwP and MA. No index structure exists for MA [8]. For EDR, we ensure uniform sampling rates through interpolation since EDR-I is the closest to EDwP in terms of robustness.

1) Online costs: Fig. 5(j) demonstrates the growth rate of querying times with $k$ (time in log-scale). TrajTree is more than an order of magnitude faster than MA and up to 5 times faster than EDR. Although the computational complexities of all three matching techniques are quadratic, MA is the slowest since it depends on 5 auxiliary functions, each of which has a quadratic computation cost. In other words, the constant factor in MA is 5 times higher. Compared to EDR, EDwP is faster since it performs dynamic interpolation through projections.

Next, we look at the growth rate of the querying time with database size. Fig. 6(a) demonstrates the result. The growth rate is sublinear for both TrajTree and EDR. EDwP is up to 5 times faster than EDR and 10 times faster than sequential scan, which establishes the efficiency of the pruning strategies. As we show later in Fig. 6(c), VPs allow us to derive a tight upper bound at the very beginning of the search process in the root node. As a result, a significant portion of the tBoxSeqs can be pruned out based on $\mathrm{EDwP}_{s u b}$. Furthermore, as the search process proceeds toward lower levels of the tree, the bounds get tighter.

Next, we study the performance dependence of TrajTree on the branching factor, which is controlled by $\theta$. As outlined in Alg. 1, $\theta$ dynamically controls the branching factor by analyzing the marginal drop in the diversity of the selected pivots. Fig. 6(b) analyzes the optimal value for $\theta$. The querying times are minimized at 0.8 , which means the optimal balance between the tightness of tBoxSeqs and the number of EDwP computations is obtained. This result influences our default choice of $\theta=0.8$.

The second parameter that impacts the performance of TrajTree is the number of vantage points. The tightness of the upper bound computed in Eq. 14 is influenced by the number of VPs distributed. We verify this tightness. Towards that goal, we introduce the notion of UB-factor.

$$
\text { UB-Factor }=\frac{\text { VP-based Upper Bound }}{k^{t h} \text { highest distance in actual } k \text {-NN }}
$$

In the best case scenario, the optimal upper bound is the $k^{t h}$ highest distance in the actual $k$-NN answer set. We therefore set the optimal upper bound as the denominator of the UBFactor. Thus, the closer the UB-Factor is to 1 , the better is the performance.

Fig. 6(c) studies the variance in UB-Factor at the root node with increase in the number of VPs. Note that at lower levels of the tree, the UB-Factor can only get tighter since TrajTree continuously refines the upper bound by evaluating only unexplored trajectories. Additionally, the density of VPs increase with decrease in tree level. In other words, Fig. 6(c) depicts the worst case scenario. As expected from a theoretical standpoint, the UB-Factors improve with increase in the number of VPs.

To further investigate the tightness of the upper bounds derived using VPs, we also study the Random UB-Factors in Fig. 6(c), denoted as Beijing Random. A Random UB-Factor is simply the UB-Factor from a random subset of $k$ trajectories in the database. Since the VP-based upper bound is derived from an approximate $k$-NN, comparison to the random UB-Factor allows us to judge the randomness in the $k$-NN approximation performed through VPs. If the $k$-NN approximation by VPs is simply a random subset of $k$ trajectories, then the difference between the VP-based UB-Factor and the random UB-Factor would be low. As can be seen in Fig. 6(c), VPs provide an UB-Factor that is significantly tighter than random and thus, efficient in pruning a large portion of the search space.

To further establish the utility of VPs, we also study the tightness of UB-Factors with increase in $k$. Fig. 6(d) presents the results. The UB-Factors are almost constant across $k$. This is a natural consequence of the fact that both the numerator and denominator of UB-Factor increases with $k$. Nonetheless, the upper bounds through VPs are more than 3 times tighter than a random selection. In addition to the above studies, we also analyzed the Spearman's rank correlation coefficient between 


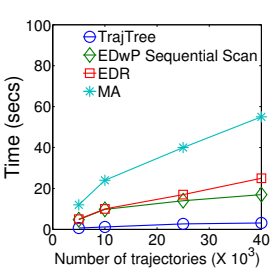

(a) Beijing

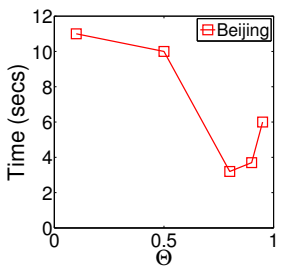

(b)

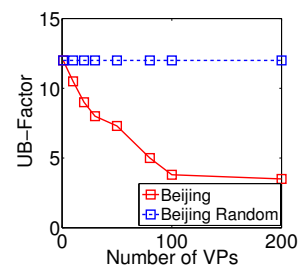

(c)

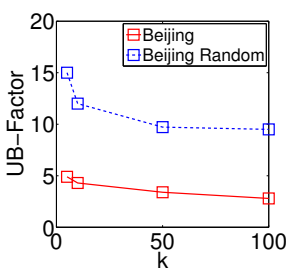

(d)

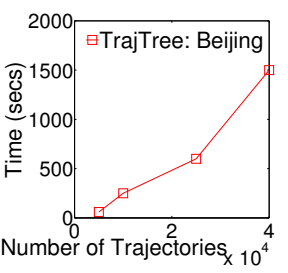

(e)

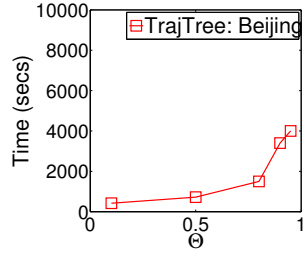

(f)

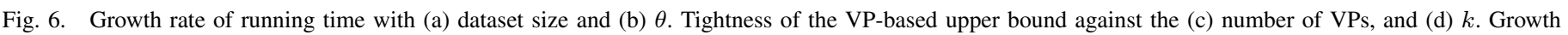
rate of index construction time with (e) database size and (f) $\theta$.

VP-based $k$-NNs and the actual answer sets. The correlation ranges between 0.78 to 0.83 across all values of $k$ in $[5,100]$.

2) Off-line Costs: First, we look at the growth rate of the index construction cost with dataset size. As can be seen in Fig. 6(e), the growth rate is smaller than quadratic but higher than a linear distribution. This result is consistent with the theoretical analysis in Sec. IV. The branching factor, regulated by $\theta$, also influences the index construction time. Fig. 6(f) studies the impact of this parameter. Consistent with the theoretical analysis in Sec. IV, the construction time increases with increase in $\theta$ as the computation cost at each level of the tree increases. This behavior is opposite to the relationship between $\theta$ and the querying time. However, since index construction is an one-time off-line computation, optimizing the querying time takes precedence.

\section{RELATED WORK}

An elaborate discussion on existing trajectory distance metrics and their weaknesses has already been done in Sec. II. Initial efforts on indexing trajectory retrieval were primarily directed towards indexing DTW [6], [20]. In subsequent works, LCSS [3], ERP [4] and EDR [5] proposed index structures for their respective distance metrics. Unfortunately, these index structures do not generalize to EDwP and thus, we develop TrajTree. Although we develop distance bounds specifically for EDwP, TrajTree generalizes the idea of bounding boxes for trajectories and can potentially be utilized for other trajectory operations. Applying bounding boxes on trajectories has also been explored by the TB-tree index [21] and SETI [22]. However, in contrast to TrajTree, both TB-tree and SETI partitions a trajectory into multiple segments, and a bounding box summarizes each of these partitions. As a result, a trajectory is distributed over various nodes in TB-tree and SETI. In TrajTree, each node is a sequence of bounding boxes that summarizes a set of trajectories. The design choice of TBtree and SETI stems from their goal of identifying database trajectories that fall within a query temporal or spatial interval. In contrast, our goal is to index similarity queries for which TB-tree and SETI cannot be used to compute a lower bound on EDwP.

\section{CONCLUSION}

In this paper, we studied the problem of matching trajectories under inconsistent sampling rates. To tackle this noise, we developed Edit Distance with Projections (EDwP), which is insulated from the choice of sampled regions, non-uniform sampling rates, and local time shifts by employing the ideas of projections and coverage. Additionally, EDwP is parameterfree. EDwP not only allows robust matching, but its ability to interpolate dynamically enables us to generalize the concept of bounding boxes for trajectories. By integrating bounding boxes with vantage points, we developed an index structure called TrajTree for fast answering of $k$-NN queries. Each vantage point provides a unique viewpoint on the trajectory database and by consolidating their views, we are able to derive tight upper bounds on the true distance. These upper bounds, in conjunction with the lower bounds derived from bounding boxes, drastically reduces the search space and provides up to an order of magnitude speed up over the state-of-the art retrieval techniques. Furthermore, extensive experiments show EDwP to be 5 times more accurate and robust than existing trajectory matching techniques.

\section{REFERENCES}

[1] "Movebank, http://www.movebank.org."

[2] M. Passe-Smith, "Exploring Local Tornado Alleys for Predictive Environmental bibtex," in ESRI User Conference, 2006.

[3] M. Vlachos, D. Gunopoulos, and G. Kollios, "Discovering similar multidimensional trajectories," in ICDE, 2002.

[4] L. Chen and R. Ng, "On the marriage of Lp-norms and edit distance," in $V L D B, 2004$.

[5] L. Chen, M. T. Özsu, and V. Oria, "Robust and fast similarity search for moving object trajectories," in SIGMOD, 2005, pp. 491-502.

[6] B. kee Yi, H. V. Jagadish, and C. Faloutsos, "Efficient Retrieval of Similar Time Sequences Under Time Warping," in ICDE, 1998.

[7] E. Frentzos, K. Gratsias, and Y. Theodoridis, "Index-based Most Similar Trajectory Search,” in $I C D E, 2007$, pp. 816-825.

[8] S. Sankararaman, P. K. Agarwal, T. Mølhave, J. Pan, and A. P. Boedihardjo, "Model-driven matching and segmentation of trajectories," in SIGSPATIAL, 2013, pp. 234-243.

[9] L.-Y. Wei, Y. Zheng, and W.-C. Peng, "Constructing popular routes from uncertain trajectories," in KDD, 2012, pp. 195-203.

[10] J. Bourgain, "On lipschitz embedding of finite metric spaces in hilbert space," Israel Journal of Mathematics, vol. 52, pp. 46-52, 1985.

[11] A. Guttman, "R-trees: A dynamic index structure for spatial searching," in SIGMOD, 1984.

[12] Y. Lou, C. Zhang, Y. Zheng, X. Xie, W. Wang, and Y. Huang, "Mapmatching for low-sampling-rate gps trajectories," in GIS, 2009.

[13] J. Yuan, Y. Zheng, C. Zhang, X. Xie, and G.-Z. Sun, "An interactivevoting based map matching algorithm," in MDM, 2010, pp. 43-52.

[14] K. Zheng, Y. Zheng, X. Xie, and X. Zhou, "Reducing uncertainty of low-sampling-rate trajectories." in ICDE, 2012, pp. 1144-1155.

[15] P. Banerjee, S. Ranu, and S. Raghavan, "Inferring uncertain trajectories from partial observations," in ICDM, 2014.

[16] J.-G. Lee, J. Han, and X. Li, "Trajectory outlier detection: A partitionand-detect framework," in ICDE, 2008, pp. 140-149.

[17] M. F. Mokbel and W. G. Aref, "Indexing historical spatio-temporal data," in Encyclopedia of Database Systems, 2009, pp. 1448-1451. 
[18] J. Yuan, Y. Zheng, X. Xie, and G. Sun, "Driving with knowledge from the physical world," in $K D D, 2011$, pp. 316-324.

[19] "Spearman's rank correlation, http://en.wikipedia.org/wiki/spearman

[20] E. Keogh and C. A. Ratanamahatana, "Exact indexing of dynamic time warping," Knowl. Inf. Syst., vol. 7, no. 3, pp. 358-386, 2005.

[21] D. Pfoser, C. S. Jensen, and Y. Theodoridis, "Novel approaches in query processing for moving object trajectories," in $V L D B, 2000$, pp. 395-406.

[22] V. P. Chakka, A. Everspaugh, and J. M. Patel, "Indexing large trajectory data sets with seti," in CIDR, 2003.

\section{APPENDIX}

\section{A. Triangular Inequality}

THEOREM 1. EDwP does not satisfy triangular inequality. Proof: Consider three trajectories $T_{1}=[(0,0),(0,1)], T_{2}=$ $[(0,0),(0,1),(0,2)]$, and $T_{3}=[(0,0),(0,1),(0,2),(0,3)]$. We ignore the timestamps since the proof is independent from them. Now, $\operatorname{EDwP}\left(T_{1}, T_{2}\right)=1 \times 1$, $\operatorname{EDwP}\left(T_{2}, T_{3}\right)=1 \times 1$, and $\operatorname{EDwP}\left(T_{1}, T_{3}\right)=2 \times 2$. Thus, $\operatorname{EDwP}\left(T_{1}, T_{2}\right)+\operatorname{EDwP}\left(T_{2}, T_{3}\right)<\operatorname{EDwP}\left(T_{1}, T_{3}\right)$.

\section{B. Proof of Lemma 3}

LEMMA 3 Consider two trajectories $T_{1}$ and $T_{2}$ such that both contain one st-segment each. If a new trajectory $T_{2}^{\prime}=$ $\left[\left[T_{2} \cdot e_{1} \cdot s_{1}, p\right],\left[p, T_{2} . e_{1} \cdot s_{2}\right]\right]$ is created by inserting an additional point $p$ on $T_{2} . e_{1}$, then $\operatorname{EDwP}\left(T_{1}, T_{2}^{\prime}\right) \leq \operatorname{EDwP}\left(T_{1}, T_{2}\right)$.

PROOF: We first define the following variables:

$$
\begin{aligned}
& p^{\prime}=p^{i n s\left(T_{1} \cdot e_{1},\left[T_{2} \cdot e_{1} \cdot s_{1}, p\right]\right)}, \\
& a=\operatorname{dist}\left(T_{1} \cdot e_{1} \cdot s_{1}, T_{2} \cdot e_{1} \cdot s_{1}\right), \\
& b=\operatorname{dist}\left(T_{1} \cdot e_{1} \cdot s_{2}, T_{2} \cdot e_{1} \cdot s_{2}\right), \\
& \Delta=\operatorname{dist}\left(p, p^{\prime}\right), \\
& d_{1}=\operatorname{dist}\left(T_{1} \cdot e_{1} \cdot s_{1}, p^{\prime}\right)+\operatorname{dist}\left(T_{2} \cdot e_{1} \cdot s_{1}, p\right), \\
& d_{2}=\operatorname{dist}\left(T_{1} \cdot e_{1} \cdot s_{2}, p^{\prime}\right)+\operatorname{dist}\left(T_{2} \cdot e_{1} \cdot s_{2}, p\right) .
\end{aligned}
$$

Fig. 7 demonstrates the variables pictorially.

In this proof, we show that the edits $\operatorname{ins}\left(T_{1} \cdot e_{1},\left[T_{2} . e_{1} . s_{1}, p\right]\right)$ (followed by $\operatorname{rep}\left(\left[T_{1} \cdot e_{1} \cdot s_{1}, p^{\prime}\right]\right.$, $\left.\left.\left[T_{2} . e_{1} . s_{1}, p\right]\right)\right)$ and $\operatorname{rep}\left(\left[p^{\prime}, T_{1} \cdot e_{1} \cdot s_{2}\right],\left[p, T_{2} \cdot e_{1} \cdot s_{2}\right]\right)$ on $T_{2}^{\prime}$ is cheaper than $\operatorname{rep}\left(T_{1} . e_{1}, T_{2} . e_{1}\right)$ on the original trajectories. Specifically,

$$
(a+b) \times\left(d_{1}+d_{2}\right)-(a+\Delta) \times d_{1}-(b+\Delta) \times d_{2} \geq 0
$$

From the construction of $T_{2}^{\prime}$, it is clear that $T_{2}^{\prime}$ 's shape is identical to $T_{2}$. Without loss of generality, we assume $b \geq a$. Due to symmetry, the proof for the opposite case follows analogously. Additionally, it is guaranteed $\nexists \Delta, \Delta>b$ and $\Delta>a$. Now, while $p$ is projected to the closest point in $T_{1} \cdot e_{1}$, in $\operatorname{rep}\left(T_{1} . e_{1}, T_{2} . e_{1}\right)$, the distance between the endpoints of the st-segments are considered. Based on this observation, we divide the proof into two cases.

Case 1: Line $p p^{\prime}$ is perpendicular to $\boldsymbol{T}_{1} \cdot e_{1}$ It is known that the shortest distance from a point to a line is its perpendicular projection. Clearly, the left hand side (LHS) of Eq. 16 is minimized if $a$ and $b$ correspond to perpendicular projections of endpoints $T_{2} \cdot e_{1} \cdot s_{1}$ and $T_{2} \cdot e_{1} \cdot s_{2}$ respectively. Fig. 7 demonstrates an instance of this case. From geometry,

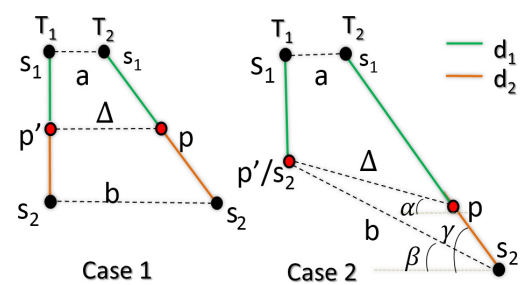

Fig. 7. Demonstrates the setup for the proof of Lemma 3. Although $T_{1}$ is assumed to be axis parallel, for arbitrary trajectories, it can always be rotated to simulate the same setup.

$b, \Delta$ and $a$ are parallel to each other and thus can be expressed as the following:

$$
\begin{aligned}
b & =a+r \times\left(d_{1}+d_{2}\right) \\
\Delta & =a+r \times\left(d_{1}\right)
\end{aligned}
$$

where $r=\frac{b-a}{d_{1}+d_{2}}$ defines the rate at which the projected distance for a point in $T_{2} \cdot e_{1}$ grows. Therefore, LHS of Eq. 16 equals:

$$
=a d_{2}+\left(a+r\left(d_{1}+d_{2}\right)\right) d_{1}-\left(a+r \times d_{1}\right)\left(d_{1}+d_{2}\right)=0
$$

Case 2: Line $p p^{\prime}$ is not perpendicular to $T_{1} \cdot e_{1}$ This case arises when $T_{1} . e_{1}$ does not contain the point that corresponds to the perpendicular projection of $p$. The non-optimality of the projected distance is maximized if $p$ is projected to one of the endpoints of $T_{1} \cdot e_{1}$. In such a case, it is guaranteed that either $a$ or $b$ is not a perpendicular projection either. Without loss of generality, we assume that $p$ is projected to $T_{1} \cdot e_{1} \cdot s_{2}$, which in turn guarantees that $b$ is the non-perpendicular projection. More precisely, $p^{i n s\left(T_{1} \cdot e_{1},\left[T_{2} \cdot e_{1} \cdot s_{1}, p\right]\right)}=p^{i n s\left(T_{1} \cdot e_{1}, T_{2} \cdot e_{1}\right)}=$ $T_{1} \cdot e_{1} \cdot s_{2}=p^{\prime}$. The proof for $p^{i n s\left(T_{1} \cdot e_{1},\left[T_{2} \cdot e_{1} \cdot s_{1}, p\right]\right)}=T_{1} \cdot e_{1} \cdot s_{1}$ follows analogously.

The LHS of Eq. 16 is minimized if $a$ is the perpendicular projection of $T_{2} \cdot e_{1} \cdot s_{1}$. We now introduce the following variables. Let $\gamma$ be the angle at which $T_{2} \cdot e_{1}$ moves away from $T_{1} \cdot e_{1}$. Furthermore, $\alpha$ and $\beta$ are the angles between $\Delta$ and $b$ with their corresponding imaginary perpendicular projections. Fig. 7 demonstrates each of these notations. Clearly, $\gamma \geq \beta \geq$ $\alpha$. In the triangle, $\triangle p^{\prime} p T_{2} . e_{1} . s_{2}, \angle T_{2} \cdot e_{1} \cdot s_{2} p^{\prime} p=\beta-\bar{\alpha}$, $\angle p T_{2} \cdot e_{1} \cdot s_{2} p^{\prime}=\gamma-\beta$, and $\angle p^{\prime} p T_{2} \cdot e_{1} \cdot s_{2}=180-\gamma+\alpha$. Using the law of sines, the following relationship is established.

$$
\begin{gathered}
\frac{d_{2}}{\sin (\beta-\alpha)}=\frac{\Delta}{\sin (\gamma-\beta)}=\frac{b}{\sin (\gamma-\alpha)} \\
b-\Delta=d_{2} \frac{\sin (\gamma-\alpha)-\sin (\gamma-\beta)}{\sin (\beta-\alpha)}
\end{gathered}
$$

Now, let $l_{1}=\operatorname{dist}\left(T_{2} \cdot e_{1} \cdot s_{1}, p\right)$. Therefore, from geometry,

$$
\Delta-a=l_{1} \frac{\cos (\gamma)}{\cos (\alpha)}
$$

Combining these observations, the LHS of Eq. 16 is

$$
\begin{aligned}
& =d_{1} \times(b-\Delta)-d_{2} \times(\Delta-a) \\
& =d_{1} d_{2} \frac{\sin (\gamma-\alpha)-\sin (\gamma-\beta)}{\sin (\beta-\alpha)}-d_{2} l_{1} \frac{\cos (\gamma)}{\cos (\alpha)} \\
& \geq d_{1} d_{2} \frac{\cos (\alpha) \sin (\gamma-\alpha)-\cos (\alpha) \sin (\gamma-\beta)-\cos (\gamma) \sin (\beta-\alpha)}{\sin (\beta-\alpha) \cos (\alpha)} \\
& \geq d_{1} d_{2} \frac{(\cos (\alpha)-\cos (\beta)) \sin (\gamma-\alpha)}{\sin (\beta-\alpha) \cos (\alpha)} \geq 0
\end{aligned}
$$

